\title{
Density of a Many-Fermion System in a Harmonic-Oscillator Potential.
}

\author{
J. Cugnon
}

Physics Department B5, University of Liège, Sart Tilman - B-4000 Lì̀ge 1, Belgium

O. HaROUNA

Université de Niamey, Service de Physique - B.P. 10662, Niamey, Rép. du Niger

(ricevuto il 17 Dicembre 1982)

PACS. 21.10. - General and average properties of nuclei; properties of nuclear energy levels.

Summary. - The ground-state density of a system of noninteracting fermions in a harmonic-oscillator potential well can be given in terms of the wave functions of a few single-particle states around the Fermi level.

We are going to demonstrate that the derivative of the density of a system of fermions occupying the lowest levels in a three-dimensional harmonic-oscillator well can be written in terms of a few radial wave functions. If $N$ major oscillator shells are occupied, the derivative implies only the last occupied shell and the first nonoccupied one.

In the one-dimensional case, the relation is very simple. If $F$ is the quantum number of the last occupied shell (Fermi level), and if the $\varphi_{i}$ 's are the normalized wave functions, one has

$$
a_{\mathbf{0}} \frac{\mathrm{d} \varrho(z)}{\mathrm{d} z}=-\sqrt{2(F+1)} \varphi_{F} \varphi_{F+1}
$$

In this relation, $\varrho$ is the density and $a_{0}$ is the harmonic-oscillator length

$$
a_{0}=\sqrt{\frac{\hbar}{m \omega}},
$$

$\omega$ being the oscillator frequency. The proof of relation (1) is given in ref. $\left.{ }^{1}\right)$ and is based on recurrence relations fulfilled by the harmonic-oscillator wave functions. The method cannot be extended to the three-dimensional case easily. We therefore prefer to start

(1) J. Cugnon and O. Harouna: Z. Phys. A, 301, 59 (1981). 
with the following sum rule $\left({ }^{2}\right)$ :

$$
\sum_{n}\left(E_{n}-E_{0}\right)\langle 0|\varrho(\boldsymbol{r})| n\rangle\langle n|z| 0\rangle=\left\langle 0\left|\frac{1}{2}[\varrho(\boldsymbol{r}),[H, z]]\right| 0\right\rangle
$$

In this relation, $|0\rangle$ is the ground state for the many-fermion Hamiltonian $H$, and $\varrho(r)$ is the density operator

$$
\varrho(\boldsymbol{r})=\sum_{i=1}^{A} \delta^{3}\left(\boldsymbol{r}-\boldsymbol{r}_{i}\right)
$$

Relation (3) is easily verified by expanding the double commutator. For the harmonicoscillator Hamiltonian

$$
H=\sum_{i}\left(\frac{p_{i}^{2}}{2 m}+\frac{1}{2} m \omega^{2} r_{i}^{2}\right)
$$

the r.h.s. is readily evaluated. One has

$$
\sum_{n}\left(E_{n}-E_{\mathbf{0}}\right\rangle\langle 0|\langle\boldsymbol{r})| n\rangle\langle n|z| 0\rangle=-\frac{\hbar^{2}}{2 m} \frac{\mathrm{d} \varrho}{\mathrm{d} z}=-\frac{\hbar^{2}}{2 m} \frac{\mathrm{d} \varrho}{\mathrm{d} r} \frac{1}{\cos \theta} .
$$

In the last relation, the spherical symmetry of the ground-state density has been used. The dependence upon the polar angle $\theta$ is eliminated by multiplying by $\cos \theta$ and integrating over the solid angles. One has

$$
-\frac{4 \pi}{3} \frac{\hbar^{2}}{2 m} \frac{\mathrm{d} \varrho}{\mathrm{d} r}=\sum_{n}\left(E_{n}-E_{0}\right) \int \mathrm{d} \Omega \cos \theta\langle 0|\varrho| n\rangle\langle n|z| 0\rangle .
$$

Both $\varrho$ and $z$ are one-body operators. Hence, they can connect the zero-particlezero-hole state $|0\rangle$ with the one-particle-one-hole states $|n\rangle=\left|p h^{-1}\right\rangle$ only, as is wellknown $\left({ }^{3}\right)$. The indice $p$ denotes the unoceupied states, whereas $h$ labels the singleparticle levels below the Fermi level. The transformation of the $A$-body matrix elements under consideration into one-body matrix elements is quite standard $\left({ }^{3}\right)$. Equation (7) writes

$$
-\frac{4 \pi}{3} \frac{\hbar^{2}}{2 m} \frac{\mathrm{d} \varrho}{\mathrm{d} r}=2 \sum_{n=p h^{-1}}\left(E_{n}-E_{0}\right) \int \mathrm{d} \Omega \cos \theta \psi_{p}(\boldsymbol{r}) \psi_{\tilde{h}}^{*}(\boldsymbol{r})\langle p|z| \tilde{h}\rangle,
$$

where $|\tilde{h}\rangle$ is the time-reversed of the single-particle state $|h\rangle$, and where $\psi$ is the total single-particle wave function. The factor 2 accounts for the spin $\frac{1}{2}$ of the fermions.

The rest of the proof relies on the following property of the three-dimensional harmonic-oscillator wave functions. If $n, l, m$ are the usual quantum numbers of the

(2) J. V. NoBle: Ann. Phys., 67, 98 (1971).

(3) A. BohR and B. R. Motrelson: Nuclear Structure (New York, N. Y., 1969), Vol. I, Chapt. 3. 
single-particle states, one has

$$
\left\langle n^{\prime} l^{\prime} m^{\prime}|z| n l m\right\rangle=a_{0} \delta_{m^{\prime} m}\left\{\delta_{n n^{\prime}} \delta_{l^{\prime} l+1}\left[\frac{\left(n+l+\frac{3}{2}\right)(2 l+1)}{(2 l+3)}\right]^{\frac{1}{2}} .\right.
$$

$\cdot(l 010 \mid l+10)(l m 10 \mid l+1 m)-$

$-\delta_{n^{\prime}, n+1}\left(1-\delta_{l 0}\right) \delta_{l^{\prime}, l-1}\left[\frac{(n+1)(2 l+1)}{(2 l-1)}\right]^{\frac{1}{2}}(l 010 \mid l-10)(l m 10 \mid l-1 m)+$

$+\delta_{n n^{\prime}}\left(1-\delta_{l 0}\right) \delta_{l^{\prime}, l-1}\left[\frac{\left(n+l+\frac{1}{2}\right)(2 l-1)}{(2 l+1)}\right]^{\frac{1}{2}}(l-1010 \mid l 0)$.

$\left.\cdot(l-1 m 10 \mid l m)-\left(1-\delta_{n 0}\right) \delta_{n^{\prime}, n-1} \delta_{l^{\prime}, l+1}\left[\frac{n(2 l+3)}{(2 l+1)}\right]^{\frac{1}{2}}(l+1010 \mid l 0)(l+1 m 10 \mid l m)\right\}$.

This relation may be derived by using the explicit expression of the harmonicoscillator wave functions and the recurrence relations for the Laguerre functions. This is a rather combersome method. It is preferable to use second quantization techniques in the spherical representation. We refer the interested reader to the book by MosHiNSKY $\left.{ }^{4}\right)$, which is one of the few textbooks to deal with the three-dimensional harmonic oscillator. It is interesting to note that the state $|n l m\rangle$ in the $N$-th major shell $(N=2 n+l)$ can be connected, through the operator $z$, with a state in the major shells $N^{\prime}=N \pm 1$ only. The first two terms in eq. (9) refer to $N^{\prime}=N+1$ and the last two terms correspond to $N^{\prime}=N-1$. This is a generalization of the onedimensional case, for which the position operator has nonvanishing matrix elements between adjacent states only.

Because of relation (9), the summation over $h$ in eq. (8) is restricted to the singleparticle states of the last occupied major shell $(N=F)$ and the indice $p$ runs only on the single-particle states of the first nonoccupied major shell. Consequently $E_{n}-E_{0}$ is equal to $\hbar \omega$. After some simple Racah algebra, eq. (8) can be put in the form

$$
\begin{aligned}
& a_{0} \frac{\mathrm{d} \sigma}{\mathrm{d} r}=-\frac{1}{\pi} \sum_{\substack{n, l \\
2 n+l=\xi^{\prime}}}\left\{\left(n+l+\frac{3}{2}\right)^{\frac{1}{2}}(l+1) R_{n l}(r) R_{n, l+1}(r)-\right. \\
&\left.-\left(1-\delta_{l 0}\right)(n+1)^{\frac{1}{2}} l R_{n l}(r) R_{n+\mathbf{1}, l-\mathbf{1}}(r)\right\},
\end{aligned}
$$

where $R_{n l}(r)$ is the radial wave function.

An expression similar to (10) is also valid, when a subshell is filled, like it is often the case in nuclear physics. For instance, the neutrons in the ${ }^{48} \mathrm{Ca}$ nucleus occupy the $N=0$ and $N=1$ shells and the $1 f_{\frac{7}{2}}$ subshell of the $N=2$ major shell. Then it is appropriate to consider the $j$-representation. One has

$$
\begin{aligned}
& \frac{\hbar^{2}}{2 m} \frac{\mathrm{d} \varrho}{\mathrm{d} r}=-\hbar \omega \frac{a_{0}}{4 \pi} \sum_{[n l j]}(2 j+1)\left\{\left[(2 l+1)(2 l+3)\left(n+l+\frac{3}{2}\right)\right]^{\frac{1}{2}}\left(\begin{array}{ccc}
l & 1 & l+1 \\
0 & 0 & 0
\end{array}\right) .\right. \\
& \cdot \sum_{\left\{j^{\prime}\right]}\left(2 j^{\prime}+1\right)\left\{\begin{array}{lcc}
j^{\prime} & j & 1 \\
l & l+1 & \frac{1}{2}
\end{array}\right\}\left\{\begin{array}{ccc}
j & 1 & j^{\prime} \\
-\frac{1}{2} & 0 & \frac{1}{2}
\end{array}\right\} R_{n, l+1}(r) R_{n l}(r)-
\end{aligned}
$$

(4) M. Moshinsky: The Harmonic Oscillator in Modern Physics: From Atoms to Quarks (New York, N. Y., 1969), p. 20. 


$$
\begin{array}{r}
-\left(1-\delta_{l, 0}\right)[(n+1)(2 l-1)(2 l+1)]^{\frac{1}{2}}\left(\begin{array}{ccc}
l & 1 & l-1 \\
0 & 0 & 0
\end{array}\right) \sum_{\left[j^{\prime}\right]}\left(2 j^{\prime}+1\right)\left\{\begin{array}{ccc}
j^{\prime} & j & 1 \\
l & l-1 & \frac{1}{2}
\end{array}\right\} \\
\left.\cdot\left(\begin{array}{ccc}
j & 1 & j^{\prime} \\
-\frac{1}{2} & 0 & \frac{1}{2}
\end{array}\right) R_{n l(r)} R_{n+1, l-1}(r)\right\}
\end{array}
$$

The summation over $n, l$ is restricted as before, to the values for which either the subshell $n, l+1$ or $n+1, l-1$ is partially or totally empty. The summation over $j\left(j^{\prime}\right)$ is restricted to the occupied (unoccupied) subshells. In the limit of the magic nuclei (all lowest major shells occupied) the summation over $j$ and $j^{\prime}$ in eq. (11) can be performed and the expression (10) is recovered.

Expressions (10) and (11) embody a curious property of the harmonic oscillator, since the derivative of the density (and therefore the density itself) is determined by just a few wave functions around the Fermi level. This property is of no practical usefulness, but it may have some important connection with nuclear physics. The three-dimensional harmonic-oscillator potential have been used extensively for many years in the development of the nuclear shell model. These days, more realistic potential wells like the Saxon-Woods potential, are used. However, it is well known that the corresponding single-particle wave functions are not very different from those of a harmonic-oscillator potential, provided the oscillator parameter is suitably chosen ( $\left.{ }^{3}\right)$. Hence, expressions like (11) can perhaps be extended to realistic potentials, for which it could be approximately valid. Of course, it has to be modified, since the parameter $\omega$ looses its meaning. A possible modification is

$$
\begin{aligned}
& \frac{\mathrm{d} \varrho}{\mathrm{d} r}=-\frac{1}{2 \pi} \frac{\sqrt{m}}{\hbar} \sum_{[n l j]}(2 j+1)\left\{\left[(2 l+1)(2 l+3)\left(n+l+\frac{3}{2}\right)\right]^{\frac{1}{2}}\left(\begin{array}{ccc}
l & 1 & l+1 \\
0 & 0 & 0
\end{array}\right) .\right. \\
& \cdot \sum_{\left[j^{\prime}\right]}\left(2 j^{\prime}+1\right)\left\{\begin{array}{ccc}
j^{\prime} & j & 1 \\
l & l+1 & \frac{1}{2}
\end{array}\right\}\left(\begin{array}{ccc}
j & 1 & j^{\prime} \\
-\frac{1}{2} & 0 & \frac{1}{2}
\end{array}\right)\left(\varepsilon_{n l+1 j^{\prime}}-\varepsilon_{n l j}\right)^{\frac{1}{2}} \cdot \\
& \cdot R_{n l+1 j^{\prime}}(r) R_{n l j}(r)-\left(1-\delta_{l 0}\right)[(2 l+1)(2 l-1)(n+1)]^{\frac{1}{2}} \cdot \\
& \left.\cdot \sum_{\left[j^{\prime}\right]}\left(2 j^{\prime}+1\right)\left\{\begin{array}{cccc}
j^{\prime} & j & 1 \\
l & l-1 & \frac{1}{2}
\end{array}\right\}\left(\begin{array}{ccc}
j & 1 & j^{\prime} \\
-\frac{1}{2} & 0 & \frac{1}{2}
\end{array}\right)\left(\varepsilon_{n+1 l-1 j^{\prime}}-\varepsilon_{n l j}\right)^{\frac{1}{2}} R_{n+1 l-l j^{\prime}}(r) R_{n l j}(r)\right\},
\end{aligned}
$$

where $\varepsilon_{n l j}$ is the single-particle energy and where the radial wave function $R_{n l j}(r)$ now depends upon the quantum number $j$ because of the spin-orbit interaction. We have tested the likelihood of such a relation on a specific example. We have chosen a Saxon-Woods potential with the parameters of ref. $\left.{ }^{5}\right)$. Figure 1 shows the results of the calculation for the ${ }^{40} \mathrm{Ca}$ nucleus. Relation (12) is strikingly fulfilled for $r \geqslant 3 \mathrm{fm}$, whereas departures appear for smaller values of $r$.

These considerations put some enlightment on a well-known observation first made by LiU and Brown $\left(^{6}\right)$. They draw the attention to the fact that in order to provide a good description of the excitations of nuclei, it is important to use a single-particle potential which gives a good description of the single-particle states near the Fermi energy. On the other hand, it is known that the coupling to low-lying excitations is

(5) K. Bear and P. E. Hodgson: J. Phys, G, 4, L287 (1978). 


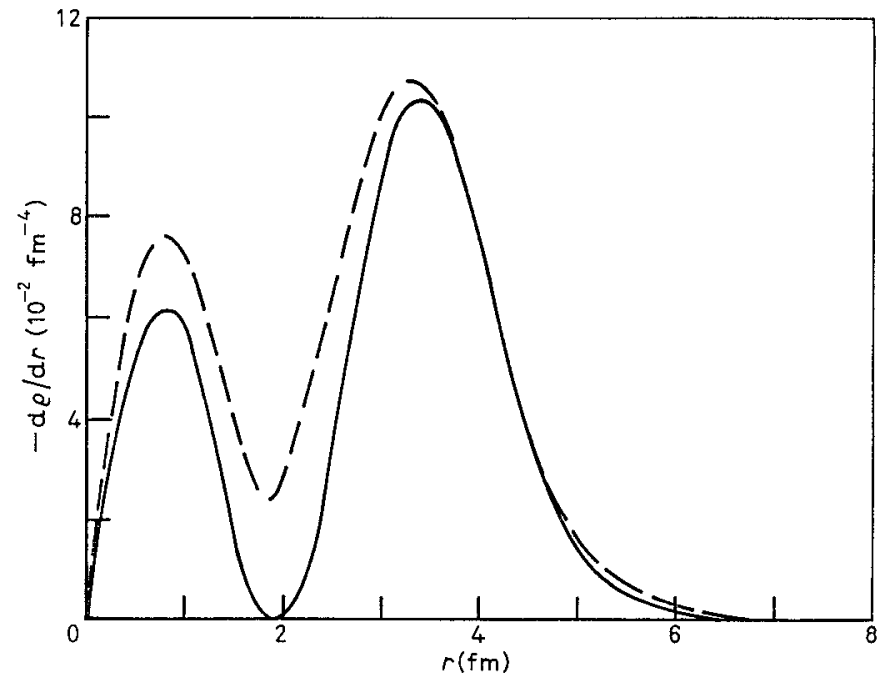

Fig. 1. - Comparison of formula (11) with the exact derivative of the nuclear density in ${ }^{40} \mathrm{Ca}$. exact, $--\rightarrow$ eq. (12).

often proportional to the derivative of the density $\left(^{7}\right)$ (multiplied by some power of $r$, according to the angular momentum of the excitation). Our result makes the observation of ref. $\left({ }^{6}\right)$ understandable. Having a good description of the single-particle states in the vicinity of the Fermi level amounts to having a good description of the transition operator.

$\left({ }^{6}\right)$ K. F. Lru and G. E. Brown: Nucl. Phys. A, 265, $385(1976)$.

(') A. Bohr and B. R. Motrelson: Nuclear Structure (New York, N. Y., 1969), Vol. II, Chapt. 6. 